\title{
Value of simultaneous assessment of cardiac functions by PET/MRI
}

\author{
Nagara Tamaki, MD, PhD, ${ }^{\mathrm{a}}$ Shigenori Matsushima, $\mathrm{MD}, \mathrm{PhD},{ }^{\mathrm{a}}$ and \\ Motoki Nishimura, $M^{a}$ \\ a Department of Radiology, Kyoto Prefectural University of Medicine, Kyoto, Japan
}

Received May 23, 2018; accepted Jun 11, 2018

doi: 10.1007/s12350-018-1341-5

See related article, pp. 1946-1957

\section{ADVANTAGES OF PET/MRI}

Hybrid positron emission tomography (PET) and computed tomography (CT) have been widely used in the clinical setting. More recently, PET and magnetic resonance imaging (MRI) have been introduced and clinically used in several centers world-wide. The integrated PET/MRI system should include special photodetectors insensitive to the magnetic field. For this purpose, either avalanche photodiodes or silicon photomultiplier technology has been used for PET signal detections. Such special requirements have also increased the cost compared to PET/CT.

The PET/MRI has several advantages and disadvantages compared to the widely used PET/CT (Table 1). Attenuation correction can be done with MRI collecting anatomical information similar to $\mathrm{CT}$. ${ }^{1}$ The most striking advantage is simultaneous acquisition of PET and MRI. Simultaneous acquisition by PET/MRI does not cause motion artifacts which are often seen with two sequential acquisitions of PET and CT using PET/CT system. In addition, PET/MRI provides less radiation burden than CT. Moreover, since MRI provides a variety of anatomical and tissue functions, including cellular and molecular studies.

PET/MRI has been applied in oncology, neurosciences, infection, inflammation, and chronic pain syndromes, in order to characterize lesion

Reprint requests: Nagara Tamaki, MD, PhD, Department of Radiology, Kyoto Prefectural University of Medicine, 465 Kajii-cho, Kawaramachi-Hirokoji, Kamigyo-ku, Kyoto, 602-8566, Japan; natamaki@med.hokudai.ac.jp

J Nucl Cardiol 2019;26:1958-61.

$1071-3581 / \$ 34.00$

Copyright (c) 2018 American Society of Nuclear Cardiology. microenvironment, such as information from liquid biomarkers (circulating tumor cells and nucleic acids) and pathology. Therefore, it can be integrated to give a more complete characterization of disease phenotype. ${ }^{2}$

While PET/MRI provides valuable functional parameters, imaging time tends to be longer as compared to PET/CT. Generally speaking, a patient throughput with PET/MRI is much less than PET/CT, and therefore, PET/MRI has been more often applied for investigative use rather than clinical use in daily practice.

\section{CARDIAC APPLICATIONS OF PET/MRI}

Regarding cardiac applications, PET has been used for viability assessment using FDG, and for stress-rest perfusion assessment using N-13 ammonia, Rb-82 or other flow/perfusion tracers. The combination of cardiac PET and cardiac MRI in a single examination should be beneficial, but is predominantly for research purposes (Table 2). Due to higher spatial resolution, CT enables precise assessment of coronary stenosis and vascular vulnerability. With use of contrast agent under pharmacological stress, stress myocardial perfusion imaging may be available by both CT and MRI. However, such information can also be acquired by PET perfusion study. MRI has advantages of higher tissue contrast resolution, lack of ionizing radiation, and is better tolerated than contrast agent. ${ }^{3}$ Actually, MRI permits assessment of perfusion abnormalities better than CT.

In addition, various tissue characterizations using suitable acquisition modes are permitted with MRI, such as tissue edema and fibrosis. Furthermore, MRI is suitable to assess global and regional ventricular functions, as well as flow dynamics. As a result, a combined study of perfusion PET and functional MRI can be obtained under a single stress study. ${ }^{4,5}$ The simultaneous acquisition of PET and MRI is one of the strong advantages of PET/MRI in the cardiovascular 
Table 1. Advantages and disadvantages of PET/ $M R I$ and PET/CT in general

\begin{tabular}{lll}
\hline & PET/MRI & PET/CT \\
\hline Morphology & + & ++ \\
Function & ++ & + \\
Molecular function & ++ & + \\
Attenuation correction & + & ++ \\
No motion artifacts & ++ & - \\
Radiation burden & + & ++ \\
Scan time & $>1$ hour & $10-30$ minutes \\
\hline
\end{tabular}

Table 2. Advantages and disadvantages of PET/ MRI and PET/CT for cardiovascular applications

\begin{tabular}{lll}
\hline & PET/MRI & PET/CT \\
\hline Coronary stenosis & - & ++ \\
Plaque assessment & + & ++ \\
Myocardial perfusion & ++ & ++ \\
LV and regional function & ++ & + \\
Flow dynamics & ++ & + \\
Viability assessment & ++ & + \\
Tissue edema & ++ & - \\
Scan time & $>1$ hour & $10-20$ minutes
\end{tabular}

applications. Moreover, MRI is commonly used for identifying tissue fibrosis using delayed contrast enhancement (DCE). Therefore, PET/MRI can be used for ischemic as well as non-ischemic cardiomyopathy in order to assess tissue viability, and cellular and molecular alterations of the myocardium.

After acute myocardial infarction, it is important to identify reversible dysfunctional area, such as hibernating myocardium. PET/MRI may identify such hibernating myocardium as an area of regional dysfunction (wall motion or thickening abnormality) on MRI with preserved glucose utilization by FDG-PET in a single acquisition. Such dysfunctional but preserved metabolic areas can be precisely identified on regional basis with simultaneous acquisition using PET/MRI. ${ }^{5,6}$ Transient dysfunctional myocardium during stress may also be identified by PET/MRI, as an area of regional dysfunction on MRI with transient decrease in stress perfusion by PET. Such transient dysfunction with stress perfusion abnormality can be identified by PET/MRI under a single stress test. Similarly, post-stress dysfunction, so called stunned myocardium, may possibly be identified with persistent regional dysfunction with recovery of perfusion with use of serial assessment after ischemia by PET/MRI. Thus, the simultaneous acquisition by PET/MRI may hold a promise to characterize precise assessment of temporal and regional changes (in another word, four dimensional changes) during ischemia as well as recovery of ischemia.

While imaging of the coronary arteries and plaque remains the domain of CT angiography, there seem to be increasing activity in the MR field. MRI holds a promise to characterize vessel wall and plaque itself. ${ }^{3,7,8}$ In the field of PET, several PET tracers have been used for atherosclerosis imaging. The most frequently used PET tracer is FDG, since increased FDG uptake is related to intense macrophage infiltration, and thus, may represent unstable plaque. ${ }^{9}$ Therefore, simultaneous acquisition of PET/MRI enables plaque location and tissue characterization in the assessment of atherosclerosis.

Based on the similar concepts, PET/MRI may hold a promise for diagnosis and assessment of various myocardial disorders, such as myocarditis and sarcoidosis. MRI is increasingly being used for infiltrative processes in acute myocarditis, using gadolinium-enhanced fast-echo T2 and T1 weighted sequences. ${ }^{3,10}$ On the other hand, FDG may accumulate in inflammatory cells, and thus, may represent acute myocarditis. PET/ MRI may enhance diagnostic accuracy of acute myocarditis and location of the inflammation.

Recently, PET/MRI has been used for tissue function for cardiac sarcoidosis. FDG-PET enables identifying active sarcoid lesions, while DCE-MRI may show myocardial fibrosis as well as cardiac function. ${ }^{11,12}$ These cellular dysfunctions identified by PET and MRI seem to be independent and both are valuable for predicting treatment effects and assessing treatment monitoring. Thus, precise tissue characterization is available by PET/MRI on regional basis for characterizing this disease.

\section{THE CURRENT PET/MRI STUDY}

Barton et al assessed the dynamic relationship between contractile function and metabolism during normoxia followed by hypoxia in pig model. ${ }^{13}$ Hypoxic stress induced a significant increase in heart rate, cardiac output, left ventricular (LV) ejection fraction (EF), and peak torsion with decrease in LV end-diastolic and endsystolic volumes assessed by MRI associated with decline in arterial $\mathrm{Sp}_{\mathrm{O}_{2}}$. Increased LV systolic function was coupled with an increase in myocardial FDG uptake (Ki) during hypoxic stress on dynamic PET study.

Their PET/MR study using continuous FDG infusion nicely showed dynamic changes in both cardiac metabolism and contractile function. They have 
maximally applied advantages of simultaneous acquisition and analysis for both function and metabolism in sequential time points of hypoxia. A serial reduction in LV-EDV without change in afterload may suggest increased LV contractility, which was coupled with a significant increase in glucose utilization during hypoxia. The rise in arterial lactate concentration during hypoxia demonstrates a global increase in anaerobic glucose metabolism.

Similar studies under serial changes with hypoxia may not be feasible in clinical condition. In addition, continuous infusion of FDG, which is quite a valuable technique for quantitative assessment, may be applied only in experimental setting, but not clinical setting. However, this study highlighted pathophysiological conditions with functional and metabolic changes under hypoxia. In addition, it may provide optimal time point for suitable study under ischemic conditions in clinical setting.

A significant reduction of FDG uptake in skeletal muscle is another attractive finding, although the authors did not discuss potential reasons for this finding. A striking reduction of blood pool of FDG might cause reduced FDG uptake in the skeletal muscle. But skeletal muscle may mainly use anaerobic glycolysis, and thus, lactate increase may not inhibit skeletal glycolysis. Striking shift from general skeletal muscle to cardiac muscle under hypoxia should be discussed.

The continuous FDG infusion system in normoxia and hypoxia over 60 minutes is attractive but should be more carefully discussed. The paper showed gradual increase in FDG myocardial uptake with decrease in FDG skeletal muscle uptake in association with plasma lactate in hypoxia. On the other hand, this model did not create steady state condition since gradual FDG uptake in the myocardium with gradual decrease in plasma FDG concentration. Therefore, it may be rather difficult to assess myocardium/plasma as well as skeletal/plasma ratios over 60 minutes.

In order to assess quantitative oxidative myocardial metabolism, C-11 acetate PET or O-15 gas may be suitable; however, these PET tracers have not been widely used, particularly not feasible under sequential analysis. Magnetic resonance spectroscopy may be another alternative for assessing myocardial hypoxic condition. However, this requires quite long time for measurement, and thus, it may not be suitable for serial assessment as the current study.

The current study nicely analyzed a number of LV strain parameters in normal and hypoxic condition. While LV volume was reduced with increase in $\mathrm{EF}$, there were no significant differences in radial or circumferential strain but only increases in peak rotation and torsion under hypoxia. Radial circumferential strains have recently been used with echocardiography and MRI in clinical setting, but these parameters may not be sensitive in animal experiments. Hypoxia may not increase cardiac contractility but increase in EF by volume reduction with increase torsion. More work has to be done in this area.

One of the inherent limitations in the current experimental study is the use of hypoxic model as hypoxia, and stress-induced ischemia is somewhat different. We do understand that hypoxia is a wellestablished experimental model to analyze global LV function with metabolic alteration. On the other hand, there are a number of animal experiments with coronary stenosis or occlusion to identify regional dysfunction with altered metabolism under pharmacological stress. ${ }^{14}$ It would be nice to see serial changes of function and metabolism using PET/MRI system under mild ischemia vs moderate to severe ischemia in order to facilitate understanding the close relationship of functional and metabolic alteration under a single stress. Also some compensatory function such as augmentation in metabolism and function in the remote area may possibly be seen with PET/MRI.

Again, we admire unique and nice experiments by the authors to suggest dynamic change of metabolism and cardiac function using PET/MRI in animal model to provide valuable information under hypoxia. PET/MRI study will surely provide us new changes for various experiments as well as clinical findings in the near future.

\section{Disclosure}

Nagara Tamaki, Shigenori Matsushima, and Motoki Nishimura have nothing to declare for this publication.

\section{References}

1. Vontobel J, Liga R, Possner M, Clerc OF, Mikulicic F, VeitHaibach P, et al. MR-based attenuation correction for cardiac FDG PET on a hybrid PET/MRI scanner: Comparison with standard CT attenuation correction. Eur $\mathrm{J}$ Nucl Med Mol Imaging. 2015;42:1574-80.

2. Bailey DL, Pichler BJ, Gückel B, et al. Combined PET/MRI: Global warming-Summary report of the 6th international workshop on PET/MRI. Mol Imaging Biol. 2018;20:4-20.

3. Rischpler C, Nekolla SG, Kunze KP, Schwaiger M. PET/MRI of the heart. Semin Nucl Med. 2015;45:234-47.

4. Kunze KP, Nekolla SG, Rischpler C, et al. Myocardial perfusion quantification using simultaneously acquired ${ }^{13} \mathrm{NH}_{3}$-ammonia PET and dynamic contrast-enhanced MRI in patients at rest and stress. Magn Reson Med. 2018. https://doi.org/10.1002/mrm. 27213. 
5. Krumm P, Mangold S, Gatidis S, et al. Clinical use of cardiac PET/MRI: current state-of-the-art potential future applications. Jpn J Radiol. 2018. https://doi.org/10.1007/s11604-018-0727-2.

6. Masuda A, Nemoto A, Yamaki T, Oriuchi N, Takenoshita S, Takeishi Y. Assessment of myocardial viability of a patient with old myocardial infarction by ${ }^{18} \mathrm{~F}$-fluorodeoxyglucose PET/MRI. J Nucl Cardiol. 2017. https://doi.org/10.1007/s12350-017-0941-9.

7. Fayad ZA, Fuster V, Fallon JT, et al. Noninvasive in vivo human coronary artery lumen and wall imaging using black blood magnetic resonance imaging. Circulation. 2000;102:506-10.

8. Kim WY, Stuber M, Bomert P, et al. Three-dimensional blackblood cardiac magnetic resonance coronary vessel wall imaging detects positive arterial remodeling in patients with nonsignificant coronary artery disease. Circulation. 2002;106:296-9.

9. Rudd JH, Warburton EA, Fryer TD, et al. Imaging atherosclerostic plaque inflammation with ${ }^{18} \mathrm{~F}$-fluorodeoxyglucose positron emission tomography. Circulation. 2002;105:2708-11.
10. Sparrow PJ, Merchant N, Provost YL, et al. CT and MR imaging findings in patients with acquired heart disease at risk for sudden cardiac death. Radiographics. 2009;29:805-23.

11. Manabe O, Oyama-Manabe N, Ohira H, Tsutsui H, Tamaki N. Multimodality evaluation of cardiac sarcoidosis. J Nucl Cardiol. 2012;19:621-4.

12. Piekarski E, Benali K, Rouzet F. Nuclear imaging in sarcoidosis. Semin Nucl Med. 2018;48:246-60.

13. Barton GP, Vildberg L, Goss K, Aggarwal N, Eldridge M, McMillan AB. Simultaneous determination of dynamic cardiac metabolism and function using PET/MRI. J Nucl Cardiol. 2018. https://doi.org/10.1007/s12350-018-1287-7.

14. Spuentrup E, Ruhl KM, Botnar RM, Wiethoff AJ, Buhl A, Jacques V, Greenfield MT, Krombach GA, Günther RW, Vangel MG, Caravan P. Molecular magnetic resonance imaging of myocardial perfusion with EP-3600, a collagen-specific contrast agent: Initial feasibility study in a swine model. Circulation. 2009;119:1768-75. 